\title{
AGB stars in binaries and their progeny
}

\author{
A. Jorissen \\ Institut d'Astronomie et d'Astrophysique, Université Libre de Bruxelles \\ C.P. 226, Boulevard du Triomphe, B-1050 Bruxelles, Belgium
}

\begin{abstract}
An AGB star in a binary system is likely to pollute its companion with carbon- and s-process-rich matter. After the AGB star has faded into an unconspicuous white dwarf, the polluted companion enters the zoo of stars with chemical peculiarities. In this paper, the progeny of AGB stars in binary systems are identified among existing spectroscopic classes (Abell 35-like, binary post-AGB, WIRRing, dwarf Ba and C, subgiant $\mathrm{CH}, \mathrm{Ba}, \mathrm{CH}, \mathrm{S}$, yellow symbiotics) and their filiation is discussed from the properties of their eccentricity - period diagrams.
\end{abstract}

\section{Introduction}

Although binarity and AGB evolution are in principle disconnected concepts (a star ought not to be member of a binary system to evolve along the AGB!), a rich woild flourishes at their contact. Its existence was first suggested by McClure et al. (1980; see also McClure 1984 and McClure \& Woodsworth 1990) in relation with the discovery of the binary nature of barium stars. Overabundances of $\mathrm{Ba}, \mathrm{Sr}$ and other heavy elements produced by the s-process are observed at the surface of these $\mathrm{G}$ and $\mathrm{K}$ giants. Their origin remained a mystery until McClure suggested that the envelopes of barium stars may have been polluted by matter accreted from a former AGB companion (being now a dim white dwarf).

That scenario raised at first several questions (Is the current companion of the barium star really a white dwarf? Where are the predicted dwarf barium stars? What is the mass transfer mode? Is wind accretion efficient enough? ...) that have now largely and satisfactorily been answered (see the recent reviews by Jorissen \& Van Eck 1998, and Jorissen et al. 1998). The mass-transfer scenario appears to be of great potential to unify the zoo of red stars with chemical peculiarities, since many such families now fit within that scenario as reviewed in Sect. 2.

\section{The mass-transfer scenario and the zoo of peculiar red stars}

Fig. 1 depicts the full evolutionary sequence involving mass-transfer from an AGB star in a binary system (phase 5). As indicated in Sect. 1, it was originally introduced to account for the chemical peculiarities exhibited by barium stars (phase 11) which could not be understood in the framework of internal nucleosynthesis in a single star. As reviewed in this section, much progress has been made since, and each phase of this binary evolutionary sequence may now be 
1. Main sequence

2. KIII, MIII

(TiO)<smiles>c1ccccc1</smiles>

○

3. $\mathrm{S}(\mathrm{Tc})$

( $\mathrm{ZrO})$

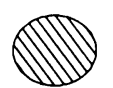

4. $\mathrm{SC}(\mathrm{Tc})$

$\left(\mathrm{ZrO}, \mathrm{C}_{2}\right)$<smiles></smiles>

RLOF or

5. $\mathrm{C}(\mathrm{Tc})$

$\left(C_{2}\right)$

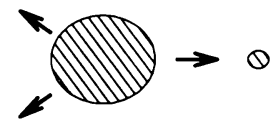

wind accretion

6. post-AGB

(1) 0

7. $\mathrm{PN}$

II

10

Abell 35-like

8. hot WD

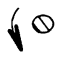

WIRRing (KVBa)

9. WD

$\circ$

$\circ$

$\circ$

○

$\circ$ dwarf $\left.\right|_{\mathrm{C}} ^{\mathrm{Ba} \text { (F strong Sr4077) }}$

subgiant $\mathrm{CH}$

giant Ba (Pop.I)

$\mathrm{CH}$ (Pop.II)

$\mathrm{S}$ or $\mathrm{C}$ (no Tc) (Pop.I)

yellow symbiotic (Pop.II)

$C(T c)(+W D)$

post-AGB (+ WD)

wide WD pair

Figure 1. Binary evolution involving mass transfer from an AGB star (phase 5). Spectral families not requiring binarity (i.e. the normal evolutionary sequence for single stars) are listed in the left column, whereas the right column identifies classes of PRS requiring binarity. Stars with excess $\mathrm{C}$ and $\mathrm{Ba}$ at their surface are represented by hatched disks 
associated with an existing class of peculiar red stars (PRS). This assignment requires both the typical chemical signature of pollution from an AGB star (i.e. excess $\mathrm{C}$ and $\mathrm{Ba}$ ) to be found in the PRS, and the PRS family to consist exclusively of binary stars.

Phase 2: Normal giants in binary systems. Catalogues of binary systems involving giant stars are provided by Boffin et al. (1993) for field stars and by Mermilliod (1996) for open-cluster stars. The pre-mass-transfer status of these systems is generally not known for sure on an individual basis (since most are SB1 systems, leaving the main-sequence or WD nature of the companion uncertain). However, the comparison of their orbital elements with those of the barium stars reveals clear differences (further discussed in Sect. 3.) which suggest that most of the G-KIII systems are indeed pre-mass-transfer systems.

Phases 3-5: $\boldsymbol{S}(\boldsymbol{T c})-\boldsymbol{S C}(\mathrm{Tc})-\boldsymbol{C}(\boldsymbol{T c})$. Binarity is not required for these PRS exhibiting lines of Tc, an element with no stable isotopes (Little et al. 1987), as they represent the normal evolutionary sequence on the AGB (Iben \& Renzini 1983).

Phase 5: Mass transfer from a $C$-rich $A G B$ star. The pollution of the companion occurs at this phase, either through wind accretion (Boffin \& Jorissen 1988; Han et al. 1995; Theuns et al. 1996; Karakas et al. 1998) or through Roche lobe overflow (RLOF) (Iben \& Tutukov 1985; Webbink 1986). The efficiency of wind accretion seems to be sufficient to account for the longest periods observed among PRS (about $30 \mathrm{y}$, see Fig. 2 and Jorissen et al. 1998), and is moreover consistent with the non-circular nature of the orbits of those postmass-transfer systems (Karakas et al. 1998). The occurrence of RLOF seems however unavoidable in the systems with circular orbits and periods shorter than a few hundred days. That question is discussed further in Sect. 3.

Phase 6: Binary post-AGB. The binary post-AGB stars identified by Van Winckel et al. (1995 and this volume) correspond most likely to this evolutionary phase. The mass functions of these SB1 systems do not always allow to distinguish between a main sequence and a WD companion. Some systems might thus correspond to phase 14 instead, although the systems with the largest mass functions $\left(>0.2 \mathrm{M}_{\odot}\right)$ very likely host main sequence companions. Most (though not all) binary post-AGB stars are very metal-deficient (Van Winckel et al. 1995), and this correlation has been interpreted as the result of re-accretion of gas depleted in refractory elements from a stable circumbinary disk (Waters et al. 1992 and this volume). Because of this chemical fractionation, the photospheric chemical content of these stars does not bear any more the signature of the third dredge-up. Whether the same (or the complementary) chemical fractionation alters as well the composition of the matter accreted by the companion (i.e. the future Ba star) is currently unknown.

Phase 7: Binary nucleus of Planetary Nebulae (Abell 35-like). Among planetary nebulae with binary nuclei, there is a class consisting of the three objects Abell $35\left(\mathrm{BD}-22^{\circ} 3467=\mathrm{LW}\right.$ Hya), LoTr 1 and LoTr 5 (HD 112313 $=\mathrm{IN}$ Com $=2 \mathrm{RE} \mathrm{J1255+255)}$ whose optical spectra are dominated by latetype (G-K) stars, but whose UV spectra indicate the presence of extremely hot $\left(>10^{5} \mathrm{~K}\right)$, hence young, WD companions (see e.g. Bond \& Livio 1990). The 
late-type star is chromospherically active and rapidly rotating. This rapid rotation is likely to result from some interaction between the binary components. Since the orbital periods are still uncertain (Jasniewicz et al. 1994; Jeffries \& Stevens 1996 and references therein; Gatti et al. 1997), the evolutionary history of these systems cannot unfortunately be identified with certainty: close binaries emerging from a common envelope phase (Bond et al. 1993) or wide binaries whose accreting star has been spun up by wind accretion (Jeffries \& Stevens 1996)? In any case, the detection of moderate overabundances of $\mathrm{Ba}, \mathrm{Y}$ and $\mathrm{Sr}$ in the G stars of Abell 35 and LoTr 5 (Thévenin \& Jasniewicz 1997; Gatti et al. 1997 do not confirm, however, that conclusion which is based on very few broad lines) indicates that these stars have been polluted by mass transfer from their former AGB companion. The similarity between these systems and the WIRRing systems described next may be in favour of a wide binary system. Furthermore, HST observations of Abell 35 with the Planetary Camera revealed a measurable extension $(0.08$ arcsec, translating into a projected separation of about $18 \mathrm{AU}$ at the minimum estimated distance of $160 \mathrm{pc}$ ) of the nucleus at $300 \mathrm{~nm}$ (Gatti et al. 1998), thus ruling out the close-binary hypothesis. Another argument favouring wide systems is provided by HD 128220, a related system consisting of a sdO and a rapidly rotating $\mathrm{G}$ star, which is the only one among those systems having a reliably determined period (872 d; Howarth \& Heber 1990).

Phase 8: WIRRing systems (rapidly-rotating $\mathrm{KVBa}+$ hot WD). The class of WIRRing stars ('Wind-induced rapidly rotating') stars has been introduced by Jeffries \& Stevens (1996) to describe a small group of rapidly rotating, magnetically-active $\mathrm{K}$ dwarfs with hot WD companions uncovered by the ROSAT Wide Field Camera or EUVE surveys (Kellet et al. 1995; Jeffries et al. 1996). From the absence of short-term radial velocity variations, it may be concluded that these systems have periods of a few months at least. Moreover, several arguments, based on proper motion, WD cooling time scale, and lack of photospheric $\mathrm{Li}$, indicate that the rapid rotation of the $\mathrm{K}$ dwarf cannot be ascribed to youth. Jeffries \& Stevens (1996) suggest that the K dwarfs in these wide systems were spun up by the accretion of the wind from their companion, when the latter was a mass-losing AGB star. The possibility of accreting a substantial amount of spin from the companion's wind has since been confirmed by Smooth Particle Hydrodynamics simulations (Theuns et al. 1996). A clear signa-

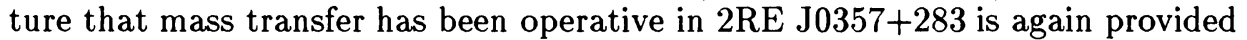
by the detection of excess barium in that star (Jeffries \& Smalley 1996).

The class of WIRRing stars may also perhaps include the barium star HD 165141, since it shares the properties of RS CVn and barium systems (Jorissen et al. 1996). The very long orbital period of the system $(P \sim 4800 \mathrm{~d}$; Fekel, private communication) forbids the barium star from having been spun up by tidal effects as is the case for RS CVn systems. This system moreover hosts the hottest WD $(T \sim 35000 \mathrm{~K})$ among barium systems (Fekel et al. 1993). The cooling time scale of the WD is about $10 \mathrm{My}$, shorter than the magnetic braking time scale of the giant star (about $100 \mathrm{My}$; see Theuns et al. 1996). The mass transfer thus occurred recently enough for the magnetic braking having not yet slowed down the giant star in a substantial way. HD 165141 is probably one of the very few barium systems where the mass transfer from the former AGB 
companion occurred when the accreting star was already a giant.

Phase 9: Dwarf Ba/C stars. About a dozen dwarf $\mathrm{C}$ stars are now known (Liebert et al. 1979, 1994; Dearborn et al. 1986; Green et al. 1991, 1992, 1994; Warren et al. 1993; Heber et al. 1993), and more may be expected from on-going dedicated surveys (MacConnell 1997). Many dC stars appear to have halo kinematics. Their binary nature has not been demonstrated in all cases.

Dwarf Ba stars (some with $\mathrm{C} / \mathrm{O}>1$ ) with orbital elements similar to those of the giant barium stars were identified by North \& Duquennoy $(1991,1992)$ and North et al. (1994) among the stars classified as FV strong Sr $\lambda 4077$ in the Michigan spectral survey. The family of $\mathrm{CH}$ subgiants identified by Bond (1974) comprises stars near and above the main sequence (Luck \& Bond 1991). The high occurrence of binaries among subgiant $\mathrm{CH}$ stars has been demonstrated by McClure (1997).

Phase 12: S(no Tc) or C(no Tc) in Pop.I, yellow symbiotics in Pop.II. The identification of the Tc-poor S stars as descendants of the barium stars was first suggested by Iben \& Renzini (1983) and Little et al. (1987), and later confirmed from the similarity of their orbital elements by Jorissen et al. (1998; see Fig. 2 and Sect. 3.). As S(no Tc) stars consist of mass-losing giants and WD companions in systems with orbital periods similar to those of the symbiotic systems, the question of the relationship between these two families must be raised. This question is actually twofold: (i) Do S(no Tc) stars exhibit some symbiotic activity? (ii) Is the barium syndrome observed among symbiotic systems? These questions have been reviewed by Jorissen (1997), and their answers may be summarized as follows: (i) Some S(no Tc) stars indeed exhibit (weak) symbiotic activity (see below); (ii) no S stars are known among 'red' symbiotics (involving $M$ giants), but all 'yellow' symbiotics (involving G or $\mathrm{K}$ giants) studied thus far are halo objects exhibiting the barium syndrome (Schmid 1994; Smith et al. 1996, 1997; Pereira et al. 1998). As the RGB is shifted towards the blue in low-metallicity populations, yellow symbiotics may be regarded as the Pop.II analogs of the S(no Tc) stars (Smith et al. 1996). The absence of $\mathrm{S}$ stars among red symbiotics is more puzzling, but most likely is a population/metallicity effect: carbon, barium and $\mathrm{S}$ stars seem difficult to form in a high-metallicity environment (Jorissen \& Boffin 1992; Jorissen \& Van Eck 1998), and carbon symbiotics are indeed frequent in the Magellanic Clouds (Mikołajewska 1997). A detailed comparison of the kinematic properties of red symbiotics and $\mathrm{S}$ (no $\mathrm{Tc}$ ) stars may shed light on that question.

A recent high-resolution survey of $\mathrm{H} \alpha$ emission among the 40 binary S(noTc) stars from Henize's (1960) sample uncovered only 2 stars with strong, broad (base width $\sim 400 \mathrm{~km} \mathrm{~s}^{-1}$ ) and double-peaked $\mathrm{H} \alpha$ emission lines (Van Eck et al., in preparation) resembling the $\mathrm{H} \alpha$ profiles of symbiotic stars (of type S3; Van Winckel et al. 1993) and of Abell 35 (Fig. 2 of Acker \& Jasniewicz 1990). The distinctive parameter responsible for the symbiotic activity in these two $\mathrm{S}$ stars is currently unknown.

The existence of two kinds of S stars (Tc and no Tc), as predicted by the mass-transfer paradigm (phases $3-5$ vs. 12 on Fig. 1), is now well established (Jorissen et al. 1993; Groenewegen 1993; Jorissen \& Van Eck 1998). Whether the same dichotomy applies to C-N stars as well is not yet firmly demonstrated, although Barnbaum (1993) found 16 Tc-poor stars in a sample of $78 \mathrm{C}-\mathrm{N}$ stars 
with Ba excess. However, their binary nature remains to be established, not an easy task given the confusion introduced by the envelope pulsation on the radial-velocity curve (Udry et al. 1998; Hinkle et al., poster contribution, this conference). Nevertheless, the recognition of this dichotomy is important for a correct interpretation of the luminosity functions of AGB stars in external systems. As shown by Van Eck et al. (1998) from HIPPARCOS data, S(no Tc) stars are on average less luminous than $\mathrm{S}(\mathrm{Tc})$ stars. They thus add a lowluminosity tail on the luminosity function of the genuine AGB S(Tc) stars. If these $\mathrm{S}$ (no Tc) [and possibly $\mathrm{C}$ (no $\mathrm{Tc}$ ) stars] are not properly removed from the luminosity functions observed in external systems, they may lead to erroneous constraints on the luminosity threshold for the occurrence of the third dredgeup in AGB stars. This danger is best illustrated by the luminosity function of carbon stars in the SMC obtained by Westerlund et al. (1995), where a formerly unrecognized low-luminosity tail extends down to $M_{\text {bol }} \sim-2$. This low-luminosity tail precisely matches the luminosity range of $\mathrm{S}$ (no-Tc) stars in the solar neighbourhood (see Figure 7 of Van Eck et al. 1998), possibly suggesting that the SMC may contain an important fraction of binary $\mathrm{C}$ (no $\mathrm{Tc}$ ) stars (see also Wood et al., this volume, for a similar conclusion)!

\section{The $(e, \log P)$ diagrams of peculiar red stars}

Fig. 2 compares the $(e, \log P)$ diagrams for the families of binaries described in Sect. 2. Orbital elements for $G-K I I I$ stars in open clusters are taken from Mermilliod (1996), for post-AGB systems (along with the sdO+G system HD 128220) from Van Winckel (this volume) and Howarth \& Heber (1990), for dwarf $B a$ and subgiant $\mathrm{CH}$ stars from North et al. (in preparation) and McClure (1997), respectively, for $B a$ and $S$ systems from Jorissen et al. (1998), and for $C H$ systems from McClure \& Woodsworth (1990). Yellow symbiotic systems displayed in Fig. 2 include TX CVn, BD-21 3873 , LT Del (=He2-467) and AG Dra, with orbital elements from various sources as listed by Smith et al. (1996).

The comparison of the $(e, \log P)$ diagrams of these various families allows to check their filiation, and to get clues on the mass transfer process.

The $(e, \log P)$ diagram of $\mathrm{G}-\mathrm{K}$ giants in open clusters differs from all the others in two clear ways: (i) many cluster giants are found with $P<200 \mathrm{~d}$, whereas such short systems are quite rare among all PRS classes; (ii) the average eccentricity at any given period is much smaller for the PRS, a fact already pointed out by Webbink (1986) for barium stars, but which now turns out to hold true for all the PRS classes. These differences between cluster giants and PRS are a clear indication that mass transfer (and possibly tidal interaction) have taken place in the PRS systems. Moreover, the non-zero eccentricities of most of the PRS systems argue against Roche lobe overflow (RLOF) as the masstransfer mode, since this mode requires the mass-loser star to fill its Roche lobe, a situation where tidal interactions will very rapidly circularize the orbit. Wind accretion is therefore preferred, as investigated by Boffin \& Jorissen (1988), Han et al. (1995) and Karakas et al. (1998). However, wind accretion by definition requires the system to remain always detached, but as discussed e.g. by Jorissen et al. (1998) and Karakas et al. (1998), this requirement may be difficult to satisfy in systems with periods as short as $300 \mathrm{~d}$. RLOF seems thus 


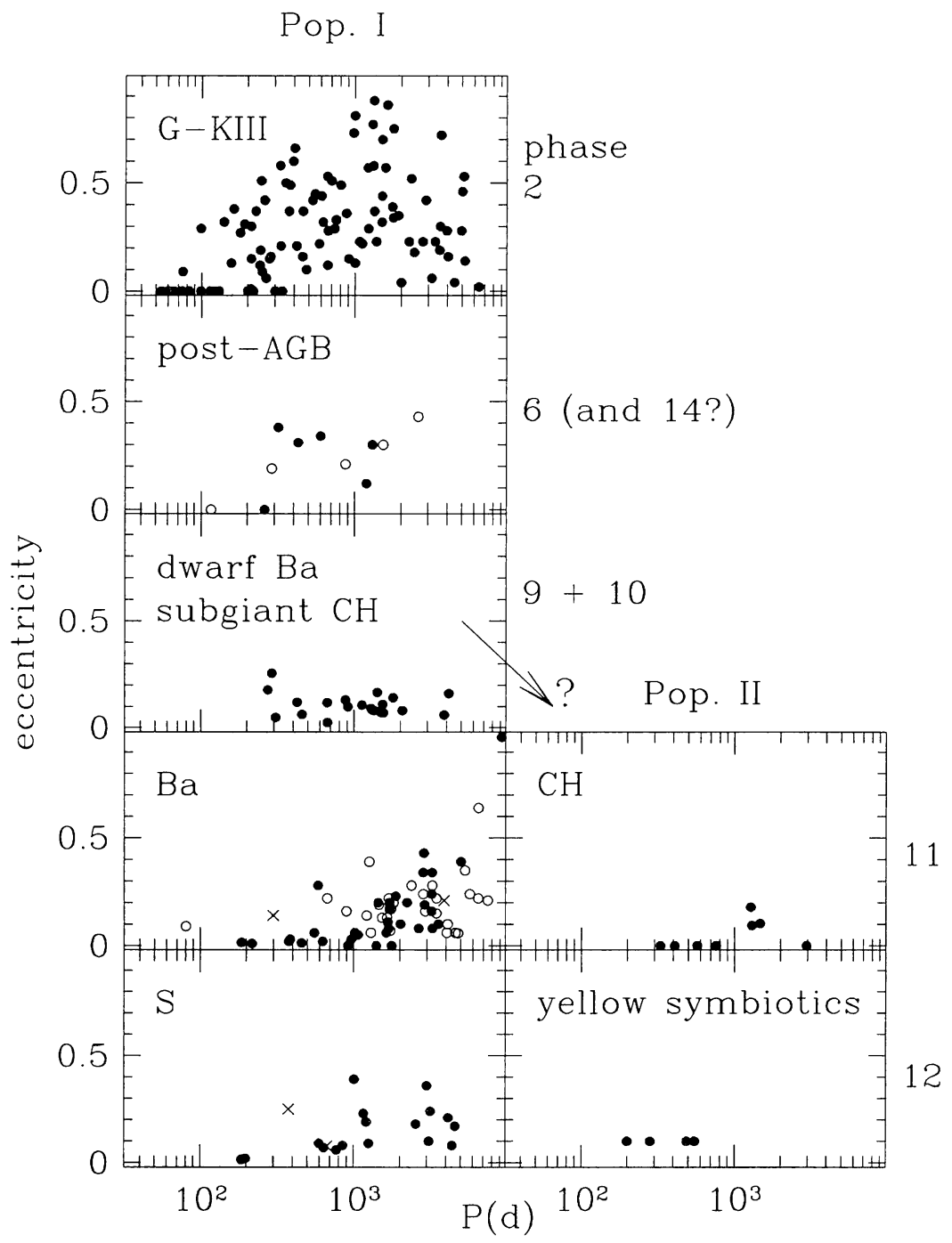

Figure 2. Comparison of the $(e, \log P)$ diagrams for the families described in Sect. 2., referred to by the phase label of Fig. 1 (written to the right of each panel). Left and right columns correspond to Pop.I and Pop.II systems, respectively. Post-AGB systems depleted in refractory elements are represented by black dots; systems not exhibiting the depletion pattern, or with no abundance data, are represented by open dots. The crosses in the $\mathrm{Ba}$ panel correspond to the two pairs of the triple system $\mathrm{BD}+38^{\circ} 118$. Ba1,2,3 (i.e. mild $\mathrm{Ba}$ ) and $\mathrm{Ba} 4,5$ (i.e. strong $\mathrm{Ba}$ ) stars are represented by open and black dots, respectively. In the $\mathrm{S}$ panel, crosses correspond to two systems with unusually large mass functions. As the eccentricities of symbiotic systems are generally poorly determined, they have all been set to 0.1 
unavoidable in these short-period systems with circular orbits (at least among Ba systems, but see below). That conclusion is troublesome, however, because RLOF from an AGB star with a deep convective envelope is generally believed to lead to a common envelope phase (at least when the mass-losing AGB star is the more massive component), and to a dramatic orbital shrinkage (e.g. Paczyński 1976; Meyer \& Meyer-Hofmeister 1979; Iben 1995). The end product of such an evolution is a cataclysmic variable with an orbital period much shorter than the shortest among PRS systems. The very existence of PRS systems with periods too short to be accounted for by the wind accretion process indicates that these systems must have avoided the dramatic fate outlined above (see also Wood, this volume). Several ways out of this channel (which sometimes does operate, though, since cataclysmic variables do exist!) have been sketched by Jorissen \& Van Eck (1998) and Jorissen et al. (1998; see also Han et al. 1995), but detailed simulations to confirm these ideas are badly needed.

The simple picture outlined above of two mass-transfer modes (RLOF for the short-period, circular orbits, and wind accretion for the long-period, eccentric orbits) that seems to emerge from the $(e, \log P)$ diagram of barium systems (after having excluded the triple system $\mathrm{BD}+38^{\circ} 118$ ), runs into problems when confronted to the $(e, \log P)$ diagrams of post-AGB, dwarf $\mathrm{Ba}$ and subgiant $\mathrm{CH}$ systems. The latter systems have significantly non-zero eccentricities, even at short periods! The picture may in fact be complicated by the following two physical processes: (i) the interaction of a binary system with a circumbinary disk (a common feature in post-AGB systems; see e.g. Waters et al., this volume) has been shown to pump the eccentricity upwards (see e.g. Lubow \& Artymowicz 1992); (ii) tidal circularization of the closest systems may occur when the $\mathrm{Ba}$ and $\mathrm{S}$ giants ascend the RGB, and account for the difference between the eccentricities of dwarf and giant $\mathrm{Ba}$ systems with the shortest periods.

A mismatch nevertheless remains for the widest systems, barium stars having larger eccentricities on average than $\mathrm{CH}$ subgiants. At this point, an observational selection effect against large eccentricities for $\mathrm{CH}$ subgiants with periods close to the time span of the monitoring cannot be ruled out as a possible origin of this mismatch (one long-period, high-eccentricity orbit is indeed pending in McClure's sample). An alternative possibility is that dwarf $\mathrm{Ba}$ and subgiant $\mathrm{CH}$ systems evolve into $\mathrm{CH}$ stars rather than into $\mathrm{Ba}$ stars. This second possibility is supported by the similarity of the eccentricity and mass function distributions of dwarf Ba stars, CH subgiants and CH stars (McClure \& Woodsworth 1990; North et al. 1994, 1998; McClure 1997), and by the often large space velocities observed for $\mathrm{CH}$ subgiants (Bond 1974), which point towards subgiant $\mathrm{CH}$ stars belonging to a population intermediate between the halo $\mathrm{CH}$ systems and the old-disk barium systems.

Finally, a fully satisfactory match is observed between $\mathrm{Ba}$ and $\mathrm{S}$ systems, which are distributed identically in the $(e, \log P)$ diagram, thus confirming the hypothesis that they only differ in terms of effective temperature. The same conclusion is reached from the similarity of their mass-function distributions (Figs. 8 and 10 of Jorissen et al. 1998).

Acknowledgments. Pierre North and Hans Van Winckel are thanked for communicating orbital elements in advance of publication. A.J. is Research Associate, F.N.R.S. (Belgium). 


\section{References}

Acker A., Jasniewicz J., 1990, A\&A 238, 325

Barnbaum C., 1993, BAAS 182, \#46.17

Boffin H.M.J., Jorissen A., 1988, A\&A 205, 155

Boffin H.M.J., Cerf N., Paulus G., 1993, A\&A 271, 125

Bond H.E., 1974, ApJ 194, 95

Bond H.E., Livio M., 1990, ApJ 355, 568

Bond H.E., Ciardullo R., Meakes M., 1993, in Planetary Nebulae, IAU Symp. 155, eds. R. Weinberger \& A. Acker, Kluwer, Dordrecht, p. 397

Dearborn D.S.P., Liebert J., Aaronson M., Dahn C.C., Harrington R., Mould J., Greenstein J.L., 1986, ApJ 300, 314

Fekel F.C., Henry G.W., Busby M.R., Eitter J.J., 1993, AJ 106, 2370

Gatti A.A., Drew J.E., Lumsden S., et al., 1997, MNRAS 291, 773

Gatti A.A., Drew J.E., Lumsden S., et al., 1997, in Planetary Nebulae (IAU Symp. 180), eds. Habing H.J., Lamers H., Kluwer, Dordrecht, p. 105

Gatti A.A., Drew J.E., Oudmaijer R.D., et al., 1998, MNRAS 301, L33

Green P.J., Margon B., MacConnell D.J., 1991, ApJ 380, L31

Green P.J., Margon B., Anderson S.F., MacConnell D.J., 1992, ApJ 400, 659

Green P.J., Margon B., Anderson S.F., Cook K.H., 1994, ApJ 434, 319

Groenewegen M.A.T., 1993, A\&A 271, 180

Han Z., Eggleton P.P., Podsiadlowski P., Tout C.A., 1995, MNRAS 277, 1443

Heber U., Bade N., Jordan S., Voges W., 1993, A\&A 267, L31

Henize K.G., 1960, AJ 65, 491

Howarth I.D., Heber U., 1990, PASP 102, 912

Iben I., Jr., 1995, Phys. Rep. 250, 1

Iben I., Jr., Renzini A., 1983, ARA\&A 21, 271

Iben I., Jr., Tutukov A.V., 1985, ApJS 58, 661

Jasniewicz G., Acker A., Mauron N., et al., 1994, A\&A 286, 211

Jeffries R.D., Smalley B., 1996, A\&A 315, L19

Jeffries R.D., Stevens I.R., 1996, MNRAS 279, 180

Jeffries R.D., Burleigh M.R., Robb R.M., 1996, A\&A 305, L45

Jorissen A., 1997, in Physical Processes in Symbiotic Stars and Related Systems, ed. J. Mikołajewska, Copernicus Foundation for Polish Astronomy, Warsaw, p. 135

Jorissen A., Boffin H.M.J., 1992, in Binaries as tracers of stellar formation, eds.

A. Duquennoy \& M. Mayor, Cambridge Univ. Press, p. 110

Jorissen A., Frayer D.T., Johnson H.R., et al., 1993, A\&A 271, 463

Jorissen A., Schmitt J.H.M.M., Carquillat J.M., et al., 1996, A\&A 306, 467

Jorissen A., Van Eck S., 1998, in The Carbon Star Phenomenon, IAU Symp. 177 , ed. R.F. Wing, Kluwer, in press

Jorissen A., Van Eck S., Mayor M., Udry S., 1998, A\&A 332, 877 
Karakas A.I., Tout C.A., Lattanzio J., 1998, MNRAS, in press

Kellet B.J., Bromage G.E., Brown A., et al., 1995, ApJ 438, 364

Liebert J., Dahn C.C., Gresham M., Strittmatter P.A., 1979, ApJ 233, 226

Liebert J., Schmidt G.D., Lesser M., et al., 1994, ApJ 421, 733

Little S.J., Little-Marenin I.R., Hagen Bauer W., 1987, AJ 94, 981

Lubow S.H., Artymowicz P., 1992, in Binaries as tracers of stellar formation,

A. Duquennoy \& M. Mayor (eds.), Cambridge Univ. Press, p. 145

Luck R.E., Bond H.E., 1991, ApJS 77, 515

MacConnell D.J., 1997, Baltic Astronomy 6, 105

McClure R.D., 1984, PASP 96, 117

McClure R.D., 1997, PASP 109, 536

McClure R.D., Fletcher J.M., Nemec J.M., 1980, ApJ 238, L35

McClure R.D., Woodsworth A.W., 1990, ApJ 352, 709

Mermilliod J.-C., 1996, in The Origins, Evolution, and Destinies of Binary Stars

in Clusters, G.F. Milone \& J.C. Mermilliod, ASP Conf. Ser. 109, p. 373

Meyer F., Meyer-Hofmeister E., 1979, A\&A 78, 167

Mikołajewska J., 1997, in Physical Processes in Symbiotic Stars and Related Systems, ed. J. Mikołajewska, Copernicus Foundation for Polish Astronomy, Warsaw, p. 3

North P., Duquennoy A., 1991, A\&A 244, 335

North P., Duquennoy A., 1992, in Binaries as tracers of stellar formation, eds.

A. Duquennoy \& M. Mayor, Cambridge Univ. Press, p. 202

North P., Berthet S., Lanz T., 1994, A\&A 281, 775

North P., Jorissen A., Mayor M., 1998, in The Carbon Star Phenomenon, IAU Symp. 177, ed. R.F. Wing, Kluwer, in press

Paczyński B., 1976, in Structure and Evolution of Close Binary Systems, IAU

Symp. 73, P.P. Eggleton, S. Mitton \& J. Whelan (eds.), Reidel, p. 75

Pereira C.B., Smith V.V., Cunha K., 1998, AJ 116, 1977

Schmid H.-M., 1994, A\&A 284, 156

Smith V.V., Cunha K., Jorissen A., Boffin H.M.J., 1996, A\&A 315, 179

Smith V.V., Cunha K., Jorissen A., Boffin H.M.J., 1997, A\&A 324, 97

Theuns T., Boffin H.M.J., Jorissen A., 1996, MNRAS 280, 1264

Thévenin F., Jasniewicz G., 1997, A\&A 320, 913

Udry S., Jorissen A., Mayor M., Van Eck S., 1998, A\&AS 131, 25

Van Eck S., Jorissen A., Udry S., Mayor M., Pernier B., 1998, A\&A 329, 971

Van Winckel H., Duerbeck H.W., Schwarz H.E., 1993, A\&AS 102, 401

Van Winckel H., Waelkens C., Waters L.B.F.M., 1995, A\&A 293, L25

Warren S.J., Irwin M.J., Evans D.W., et al., 1993, MNRAS 261, 185

Waters L.B.F.M., Trams N.R., Waelkens C., 1992, A\&A 262, L37

Webbink R.F., 1986, in Critical Observations vs. Physical Models for Close

Binary Systems, eds. K.-C. Leung \& D. Zhai, Gordon and Breach, p. 403

Westerlund B.E., Azzopardi M., Breysacher J., et al., 1995, A\&A 303, 107 